\title{
Joint density-functional theory for electronic structure of solvated systems
}

\author{
S.A. Petrosyan, Jean-Francois Briere, David Roundy, and T.A. Arias \\ Laboratory of Atomic and Solid State Physics, Cornell University
}

(Dated: November 5, 2018)

\begin{abstract}
We introduce a new form of density functional theory for the $a b$ initio description of electronic systems in contact with a molecular liquid environment. This theory rigorously joins an electron density-functional for the electrons of a solute with a classical density-functional theory for the liquid into a single variational principle for the free energy of the combined system. A simple approximate functional predicts, without any fitting of parameters to solvation data, solvation energies as well as state-of-the-art quantum-chemical cavity approaches, which require such fitting.
\end{abstract}

$A b$ initio electron density-functional methods have proved a computationally efficient and accurate tool for the exploration of a wide range of issues in condensedmatter physics and chemistry. (See, for instance ${ }^{1,2}$.) However, application of this approach is largely limited to the solid-state, gas phase chemistry, or to surface chemistry in high vacuum environments, with practical problems involving liquid environments largely out of reach. The reason for this unfortunate limitation is that proper description of the effects of water demands not only the inclusion of many solvent molecules but also thermodynamic sampling of many configurations of those molecules so as to properly capture the structure and response of the liquid over experimental length and time scales. Thus, the applicability of such approaches to the vast array of problems involving liquid environments, from liquid fuel-cell research to biochemistry, is severely curtailed.

In response, much effort (many thousands of articles and a large number of reviews,$\frac{3,4,5,6,7}{,}$ and others, in the last decade alone) has been dedicated to the development and application of a large number of different "continuum" solvation models, in which the details of the molecular aqueous environment are replaced by a continuum description. More recently, Dzubiella et al $\underline{\underline{8}}$ developed a continuum model for water where hydrophobic, dispersion and electrostatic energy terms are written as functionals of the exclusion volume. The plethora of models evidences the importance of the problem, but the lack of a consensus underscores that no truly satisfactory method has been found.

The weaknesses of the current state of the art in continuum solvation approaches arises from their basic structure. As Ref. $\underline{7}$ and the other reviews cited above describe, such approaches generally divide the free energy of solvation into a number of contributions, typically cavitation (formation of the solvent-solute interface), dispersion (long-range electron correlations), repulsion (shortrange electron overlap effects), and electrostatic (reorientational and polarization screening in the solvent). Work then proceeds to develop models for each of these terms separately. The models for these terms generally require a cavity shape, which is most usually defined by spheres representing atoms or functional groups, where the sphere radii are determined by fits of the final results to a large empirical database of solvation energies ${ }^{3}$.
Finally, to account for nonlinear saturation effects near solutes, an intermediate dielectric constant is often used in a shell around the solute as a buffer between the solute and bulk medium, as in Ref $\underline{\underline{9}}$ for example. Despite the successes of this method, the ad hoc separation of physical effects (all originating ultimately from the underlying quantum and statistical mechanics) and the empirical fitting to precisely the class of quantities of interest limits the reliability of the predictions of these models. This is especially true for applications to new classes of chemical species or to situations outside of the fitting database, such as to study liquid phase surface catalysis.

The new approach which we propose to pursue here suffers none of the aforementioned difficulties. We first prove a theorem which shows that the thermodynamics of a system and its electrons (solute) in equilibrium with a liquid environment (solvent) can be described rigorously in terms of a joint density-functional theory (JDFT) between the electrons in the system and the molecules comprising the solvent. The physics of the equilibrium between a solute and a solvent (cavity formation, dielectric screening, dispersion and repulsion) are then all determined in a single variational principle. Maintaining the first principles nature of density-functional theory, this new approach thus requires no artificial separation of contributions, no ad hoc definitions of cavity shapes, and no empirical fitting of parameters to experimental solvation energies. As shown below, even a preliminary implementation of this new approach gives results which are competitive with state-of-the-art continuum solvation models, even without direct fitting to any solvation data. This suggests that further refinements will result in a new, efficient and predictive approach to electronic structure in the presence of liquid environments.

Joint density-functional theory - A straightforward combination of Mermin's non-zero temperature formulation of density-functional theory ${ }^{10}$ with Capitani et al.'s extensions of the zero-temperature theory to include nuclear degrees of freedom 11 leads to the following, exact variational principle for the total thermodynamic free energy of an electron-nuclear system in a fixed external electrostatic potential $V(r)$

$$
A=\min _{n_{t}(r),\left\{N_{i}(r)\right\}}\left\{F\left[n_{t}(r),\left\{N_{i}(r)\right\}\right]+\right.
$$




$$
\left.\int d^{3} r V(r)\left(\sum_{i} Z_{i} N_{i}(r)-n_{t}(r)\right)\right\},
$$

where $n_{t}(r)$ is the thermally and quantum mechanically averaged total single-particle number density of electrons, $N_{i}(r)$ is the likewise averaged density of the nuclear species $i$ (of atomic number $Z_{i}$ ), and $F$ is a universal functional. (Here, as throughout this work, we employ atomic units, in which Planck's constant and the mass and charge of the electron all have value unity, $\hbar=m_{e}=e=1$.) The universality properties of the functional $F$ may be seen directly from its construction within Levy's constrained search procedure ${ }^{12}$,

$$
\begin{aligned}
& F\left[n_{t}(r),\left\{N_{i}(r)\right\}\right] \equiv \\
& \min _{\hat{\rho} \rightarrow\left[n_{t}(r),\left\{N_{i}(r)\right\}\right]} \operatorname{Tr}\left(\hat{\rho} \hat{H}+k_{B} T \hat{\rho} \ln \hat{\rho}\right),
\end{aligned}
$$

where $k_{B} T$ is the thermal energy, $\hat{H}$ represents all interactions among and the kinetic energy of the electrons and nuclei, $\hat{\rho}$ is the full quantum-mechanical density matrix for the electron and nuclear degrees of freedom, and the minimization is carried out over only those $\hat{\rho}$ which lead to the given densities $n_{t}(r)$ and $\left\{N_{i}(r)\right\}$. From this construction it is clear that $F$, like $\hat{H}$ from which it derives, is independent of the external potential $V(r)$ and depends only upon the identities of the nuclear species $i$ (and, implicitly, upon the temperature $T$ ), as Capitani et al. 11 found previously for the case of $T=0$.

To employ the variational principle Eq. (1) in the study of a system to be treated explicitly in contact with a solvent environment, we take the nuclear species $i$ to be those comprising the environment (solvent) and the potential $V(r)$ in Eq. (11) to be that arising from the nuclei of the explicit system (solute),

$$
V(r) \equiv \sum_{I} Z_{I} /\left|r-R_{I}\right|
$$

which we take to sit at fixed locations $R_{I}$ with atomic numbers $Z_{I}$. Note that, although we employ a BornOppenheimer approximation for the nuclei of the explicit system, at this stage the treatment of the nuclear species of the environment in Eq. (2) is fully quantum mechanical. Thus, Eqs. (12) account for all zero-point motion effects associated with lighter nuclear species in the solvent, such as may be associated with the protons in liquid water or with the helium atoms in superfluid helium when used as a solvent.

Although Eqs. (12) give a rigorous continuum treatment of the environment nuclei, the variational principle Eq. (11) is ultimately impracticable because it requires explicit treatment of all of the electrons, including those associated with the environment. We thus "integrate out" the electrons associated with the environment by writing $n_{t}(r)=n(r)+n_{e}(r)$, where $n_{e}(r)$ is the electron density associated with the environment and $n(r)$ is the electron density associated with the system in contact with that environment. We then perform the minimization over all allowable $n_{e}(r)$, and finally perform the minimization over all allowable $n(r)$. For this purpose, we define the sets of allowable $n_{t}(r), n_{e}(r)$ and $n(r)$ to be all Nrepresentable functions satisfying the criteria of Gilbert 13 and integrating to the appropriate number of electrons for the respective system. Because all thus defined $n_{t}(r)$ can be constructed as the sum of some allowable $n_{e}(r)$ and some allowable $n(r)$ and because all such allowable $n_{e}(r)$ and $n(r)$ sum to an allowable $n_{t}(r)$, this procedure is guaranteed to recover the final free energy in Eq. (1). Thus, we have

$$
\begin{aligned}
A= & \min _{n(r),\left\{N_{i}(r)\right\}}\left\{G\left[n(r),\left\{N_{i}(r)\right\}, V(r)\right]\right. \\
& \left.-\int d^{3} r V(r) n(r)\right\}
\end{aligned}
$$

where $V(r)$ is defined above in Eq. (3) and where

$$
\begin{aligned}
& G\left[n(r),\left\{N_{i}(r)\right\}, V(r)\right] \equiv \\
& \min _{n_{e}(r)}\left\{F\left[n(r)+n_{e}(r),\left\{N_{i}(r)\right\}\right]\right. \\
& \left.+\int d^{3} r V(r)\left(\sum_{i} Z_{i} N_{i}(r)-n_{e}(r)\right)\right\}
\end{aligned}
$$

is universal in the sense that its functional form, like $F$ from which it derives, depends only on the nature of the solvent and, implicitly, the temperature, and that its dependence on the solute is only through the electrostatic potential of the nuclei in $V(r)$. The choice to separate the interaction $-\int d^{3} r V(r) n(r)$ in Eq. (4) from the definition of $G$ limits the interactions which the unknown functional $G$ must describe, easing the task of finding good approximations. Note, for instance, that with this choice $V(r)$ in Eq. (5) now couples to a neutral charge distribution, thereby limiting to the greatest extent possible the dependence of $G$ on $V(r)$.

Eq. (4) gives the exact free energy and exact configuration of the solvent $\left\{N_{i}(r)\right\}$. However, care must be taken in the interpretation of the $n(r)$ which yield the minimum value. The indistinguishability of electrons implies that there can be no fundamentally meaningful assignment of electrons as belonging either to the environment or to the system, and thus no exact formulation can give a unique result for $n(r)$ without some additional prescription. Indeed, for the exact $\left\{N_{i}(r)\right\}$ and any $n(r)$ which integrates to the correct number of electrons and is everywhere less than the exact solution $n_{t}(r)$ so that $n_{e}(r)=n_{t}(r)-n(r)$ is allowable in the above sense, the minimization in Eq. (5) will find $n_{e}(r)=n_{t}(r)-n(r)$ and thus ultimately produce the exact value for $A$. There is thus a large set of $n(r)$ which yield the minimum value in Eq. (4), and the variational principle embodied in Eqs. (45) satisfies the fundamental condition of not enforcing any particular, arbitrary decomposition of the total electron density into solvent and environment contributions. 
In practice, however, we expect approximations to Eq. (5) to break the above degeneracy and to pick out a unique solution. The standard pseudopotential method, which replaces the effects of the nuclei and (relatively) inert core electrons of a solid or molecule with an effective or "pseudo-" potential ${ }^{\underline{1}}$, is in fact an example of an approximation which tracks only a portion of the total electron density and provides results approaching chemical accuracy while suffering no pathologies related to the underlying degeneracy of an apportionment of electrons between two subsystems.

The existence and reliability of so called "molecular pseudopotential" Hamiltonians ${ }^{14,15,16}$ implies the existence of reliable approximations to Eq. (5) which pick out a unique solution for $n(r)$. Such pseudopotential Hamiltonians replace the effects of the nuclei and electrons of a collection of molecules on the electrons of an external system (solute) with an effective potential $V_{\left\{R_{i}\right\}}(r)$, which depends explicitly on the locations of the molecular nuclei $\left\{R_{i}\right\}$. Such Hamiltonians have proved to be quite accurate. Using them, for instance, Vaidehi et al. find the solvation energy of $\mathrm{Li}^{+}$to within $0.6 \mathrm{kcal} / \mathrm{mole}$, and Kim, Park and co-workers find results for total energies with an accuracy acceptable for the study of the problem of an excess electron solvated in water.

Formulating the exact thermodynamics of such Hamiltonians with the same approach that leads to Eq. (1) gives directly the principle in Eq. (4), but now with

$$
\begin{aligned}
& G\left[n(r),\left\{N_{i}(r)\right\}, V(r)\right] \equiv \\
& \min _{\hat{\rho} \rightarrow\left[n(r),\left\{N_{i}(r)\right\}\right]} \operatorname{Tr}\left(\hat{\rho} \hat{H}_{\left\{R_{i}\right\},\left\{R_{I}\right\}}+k_{B} T \hat{\rho} \ln \hat{\rho}\right),
\end{aligned}
$$

where $n(r)$ represents the electron density associated with the solute alone and $\hat{H}_{\left\{R_{i}\right\},\left\{R_{I}\right\}}$ represents the internal electron gas Hamiltonian for the solvent electrons alone, the interaction of these electrons with the molecular pseudopotential $V_{\left\{R_{i}\right\}}(r)$ and a model potential function $U\left(\left\{R_{i}\right\},\left\{R_{I}\right\}\right)$ describing the interactions among the environment molecules and the interaction between those molecules and the nuclei of the solute through the electrostatic potential $V(r)$ defined in Eq. (3). Because the electrons have been already apportioned between the solute and the solvent during the construction of $\hat{H}_{\left\{R_{i}\right\},\left\{R_{I}\right\}}$, the functional $G$ in Eq. (6) represents an example of an approximation to Eq. (5) which is both reliable and free of any potentially pathological issues associated with degenerate solutions for $n(r)$.

With the functional dependence of $G$ established in Eq. (44), we next separate out known components and leave an unknown part to be approximated,

$$
\begin{aligned}
& G {\left[n(r),\left\{N_{i}(r)\right\}, V(r)\right] \equiv A_{K S}[n(r)] } \\
& \quad+A_{l q}\left[\left\{N_{i}(r)\right\}\right]+U\left[n(r),\left\{N_{i}(r)\right\}, V(r)\right],
\end{aligned}
$$

where $A_{K S}[n(r)]$ is standard universal Kohn-Sham electron-density functional of the explicit system when in isolation, $A_{l q}\left[\left\{N_{i}(r)\right\}\right]$ is the "classical" densityfunctional for the liquid solvent environment when in isolation, and $U\left[n(r),\left\{N_{i}(r)\right\}, V(r)\right]$, defined formally and exactly as the difference between the exact functional and the sum of the two former functionals, is a new functional describing the coupling between the systems. The new functional $U[n(r), N(r), V(r)]$ has the same universality properties as the functional $G$ from which it derives.

Construction of approximate functionals - Working with (77) requires an approximate functional $A_{l q}\left[\left\{N_{i}(r)\right\}\right]$ for the bulk liquid. For water this is an active area of research $17,18.19$. To describe water in our preliminary implementation, we built on the ideas and perspective of Sun 17 . We first imagine minimizing over the proton density so that a single field remains, the density $N(r)$ of the oxygen nuclei, which one may view as the "molecular density" as determined by taking the oxygen nucleus to define the location of each molecule. Our version of the resulting functional then takes the form

$$
\begin{aligned}
& A_{l q}[N(r)]= \\
& A_{i d}[N(r)]+\int N(r)\left[\epsilon_{h s}(N(r))-a N(r)\right] d^{3} r \\
& -b \int N(r)\left[\int g_{\sigma}\left(r-r^{\prime}\right) N\left(r^{\prime}\right) d^{3} r^{\prime}-N(r)\right] d^{3} r .
\end{aligned}
$$

The first term in Eq. (8), $A_{i d}[N(r)]=$ $k_{B} T \int N(r)\left(\ln \left(N(r) \lambda^{3}\right)-1\right) d^{3} r$ is the analytically exact functional for the ideal gas, where $k_{B} T$ is the thermal energy and $\lambda$ is the thermal de Broglie wave length of the solute molecules. In the second term, $\epsilon_{h s} \equiv k_{B} T\left((3 / 2)\left((1-\eta)^{-2}-1\right)-\ln (1-\eta)\right)$ is the Percus-Yevick approximation ${ }^{20}$ for the free energy per particle of a system of hard spheres of diameter $d$ over and above that of the ideal gas, where $\eta \equiv\left(\pi d^{3} / 6\right) N(r)$ with $d$ being the sphere diameter (fit to experimental parameters below). (In retrospect, this work should have employed the Carnahan-Starling approximation, which more accurately describes hard spheres and represents little or no computational overhead. For the range of parameters relevant here, the two approximations agree to within about $4 \%$, and so we expect little significant change to the final results.) The constant $a$ in this second term describes the cohesive tendency between molecules that holds the fluid together. The third term is written so that it is non-zero only if $N(r)$ is not constant, where $b$ is a coupling constant and $g_{\sigma}(r) \equiv \exp \left(-r^{2} / 2 \sigma^{2}\right) /\left(2 \pi \sigma^{2}\right)^{3 / 2}$ is a normalized Gaussian describing the range of non-local behavior.

The first two terms in Eq. (8) capture the properties of the bulk fluid. The two parameters $a$ and $d$ in these terms were adjusted to reproduce the equilibrium density and bulk modulus of water with results summarized in Table I. For the final term which describes inhomogeneities, the non-local coupling strength $b$ and the range $\sigma$ were adjusted to reproduce the macroscopic surface tension of water $\gamma$ and the approximate location $R_{b}$ of the "bend" (as measured by the point of maximum downward curvature) of the surface tension versus sphere radius prediction of the molecular dynamics data of ten Wolde and co-workers ${ }^{21}$. Table \ summarizes these results as well. 


\begin{tabular}{l||cc|cc} 
parameters & $\begin{array}{c}\mathrm{a} \\
\left(\frac{\mathrm{J} \mathrm{m}^{3}}{\mathrm{~mole}^{2}}\right)\end{array}$ & $\begin{array}{c}\mathrm{d} \\
(\mathrm{nm})\end{array}$ & $\begin{array}{c}\mathrm{b} \\
\left(\frac{\mathrm{J} \mathrm{m}^{3}}{\mathrm{~mole}^{2}}\right)\end{array}$ & $\begin{array}{c}\sigma \\
(\mathrm{nm})\end{array}$ \\
\hline values & 0.3944 & 0.2918 & 0.1561 & 0.4388 \\
\hline \hline properties & $\begin{array}{c}N_{b} \\
\left(\mathrm{~kg} / \mathrm{m}^{3}\right)\end{array}$ & $\left.\begin{array}{c}B \\
\mathrm{GPa}\end{array}\right)$ & $\begin{array}{c}\gamma \\
\left(\mathrm{mJ} / \mathrm{m}^{2}\right)\end{array}$ & $\begin{array}{c}R_{b} \\
(\mathrm{~nm})\end{array}$ \\
\hline this work & 998.3 & 2.184 & 71.93 & 3.70 \\
experiment $(*)$ & 998.2 & 2.190 & 72.75 & $\sim 4$
\end{tabular}

TABLE I: Fit parameters from (8) with comparisons between model and experimental results (at standard conditions of $20^{\circ} \mathrm{C}$ and atmospheric pressure. (*) The value for $R_{b}$ is theoretical $^{21}$. (See text.)

We emphasize that although some of the parameter fits in this preliminary formulation are empirical, they do not involve any direct fit to molecular solvation free energies.

For the coupling functional $U[N(r), n(r), V(r)]$ in (7), we proceed by dividing it into two parts: long-range dielectric screening $\Delta U_{s c}[n(r), V(r)]$ capturing the tendency of the molecules in the liquid to be found in orientations and polarization states that tend to screen longrange electric fields, and a short-range electron-overlap interaction $\Delta U_{e l}[N(r), n(r)]$. The long-range screening depends only on the electrostatics of the solute and so depends only on its electron density $n(r)$ and the nuclear electrostatic potential $V(r)$. The electron-overlap contribution depends upon contact between the solvent molecules and the solute electron density and so to some approximation depends only upon these two densities.

The lowest-order form for the electronic coupling between the liquid and the solute which is compatible with translational and rotational symmetry is then

$$
\Delta U_{e l}[N(r), n(r)]=\int d^{3} R \int d^{3} r n(r) V_{p s}(r-R) N(R) .
$$

Such a lowest-order coupling is a reasonable starting point as the overlap is small. With this form, the convolution kernel $V_{p s}$ plays the role of the average potential which an electron at point $r$ feels from a water molecule at point $R$, similar to the "molecular" pseudopotential of the type introduced by Cho and coworkers in the different context described above $\frac{15}{2}$. The main difference between this potential and that of Ref $\frac{15}{5}$ is that in the present, preliminary formulation, the pseudopotential contains no information about the orientation of the molecules and so represents some sort of orientational average. To optimize numerical convergence, we choose to fit $V_{p s}(r)$ to the sum of two origin-centered Gaussians of adjustable width and amplitude for a total of four adjustable parameters,

$$
V_{p s}(r)=A_{1} e^{-\frac{r^{2}}{2 \sigma_{1}^{2}}}+A_{2} e^{-\frac{r^{2}}{2 \sigma_{2}^{2}}}
$$

We then adjusted these parameters to reproduce the orientationally averaged interaction of a water molecule

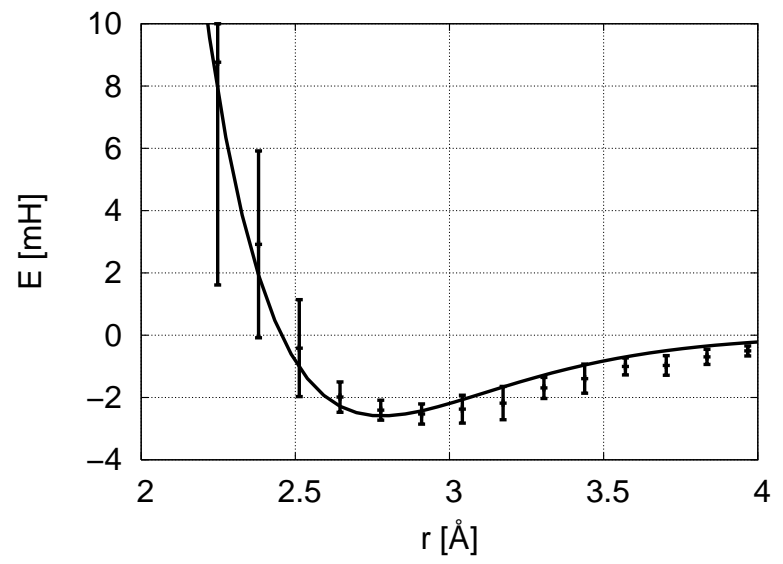

FIG. 1: Comparison of energy of interaction of water molecule with an atom of neon as a function of neon-oxygen distance: results from orientation independent pseudopotential (solid curve), orientally averaged $a b$ initio data (centers of error bars), typical $(1 \sigma)$ range of values as a function of orientation (range of error bars).

with an atom of neon as a function of distance. Figure1 summarizes the results. The parameters that we found are

$$
A_{1}=0.0765 ; \sigma_{1}=2.045 ; A_{2}=-0.065 ; \sigma_{2}=2.165 .
$$

With this simple form, we were able to reproduce the average interaction to within 1 millihartree $(\sim$ $0.63 \mathrm{kcal} / \mathrm{mole}$ ) for all distances beyond $2 \AA$. (Smaller distances are not very relevant as the interaction becomes very repulsive. For comparison, at room temperature, $k_{B} T=0.93 \mathrm{mH}$.) Here, there is no fitting to empirical data, and the pseudopotential is truly $a b$ initio in the same sense as an electron pseudopotential. As such, Vps does not afford an opportunity to fold solvation data into the construction of our functional.

Next, the screening term depends upon the long-range electrostatic potential of the solute. We take it to be the change in electrostatic energy of this potential in the presence of a linear, non-local dielectric function of the form

$$
\epsilon\left(r, r^{\prime}\right) \equiv \delta\left(r-r^{\prime}\right)+\frac{4 \pi \chi_{b}}{N_{b}^{2}} N(r) f\left(r-r^{\prime}\right) N\left(r^{\prime}\right),
$$

where $\delta(r)$ is the Dirac-delta function, $f(r)$ is a shortranged function integrating to one, and $N_{b}$ and $\chi_{b}$ are the density and static dielectric polarizabilities, respectively, of the bulk liquid. This choice ensures a smooth transition from the dielectric constant of the bulk to that of vacuum over the length-scale of $f(r)$. The choice to include both $N(r)$ and $N\left(r^{\prime}\right)$ is motivated both by the need for $\epsilon\left(r, r^{\prime}\right)$ to be symmetric and by the notion that the response at point $r$ to a field at point $r^{\prime}$ depends on the density of molecules at both locations. The connecting function $f(r)$ was chosen to be a Gaussian of width $\sigma=2.25 \mathrm{bohr}=\sim 1.190 \AA$, somewhat larger than the $\mathrm{O}-\mathrm{H}$ 


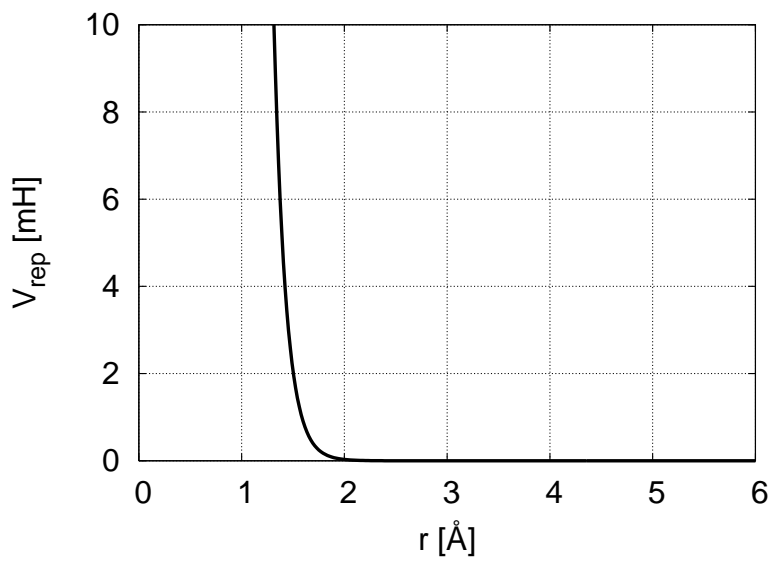

FIG. 2: Short-ranged repulsive potential added to prevent collapse of liquid density $N(r)$ into the strong electric fields within the atomic cores. Once prevention of this artificial collapse is achieved, the final results are insensitive to the choice of this potential.

bond distance in water. The motivation for using this value of $\sigma$ is that, at lower values, dielectric screening at short length-scales is so effective that the system may lower its energy by bringing fluid density $N(r)$ into the atomic cores and the system thus becomes numerically unstable. We stress, however, that once stability was achieved, the final results were not sensitive to the choice of this parameter (typically $10 \%$ variation in the solvation energy over the range $\sigma=1.5 \mathrm{bohr}$ to $\sigma=2.5 \mathrm{bohr}$ ) and that this parameter was in no way adjusted to reproduce experimental solvation energies. In the future, a direct description of the electric polarization in terms of the orientational state of the solvent would capture dielectric effects directly and remove the need for constructing such a simple, ad hoc model.

Finally, in conjunction with a nonlocal dielectric response, a term must be added to the energy functional to help prevent the aforementioned numerical issues associated with penetration of the solvent density into the cores of the atoms. To avoid this, we added a short-ranged repulsive potential of the form $\int V_{r e p}(r) N(r) d^{3} r$ inside the atomic cores to prevent the overlap of the solvent with the nuclei, where $V_{\text {rep }}(r)$ is taken to be a rapidly decaying exponential function (rounded within $\sim 0.25 \AA$ of the origin) of constant $8.44 \AA^{-1}$, leading to an apparent hard wall at thermal energy scales near $1.5 \AA$ (Figure 2). Again, once the system is numerically stable, the results are not sensitive to the form of the repulsion (typically $1 \%$ changes in solvation energy) so long as the repulsion effectively cuts off before the natural point of closest approach of the solvent at $\sim 2 \AA$. (See Figure 3 and the corresponding discussion below.)

Results and Conclusions - The meaningful physical quantities predicted by joint-density functional theory include not only the free energy $A$ but also the liquid density $N(r)$. Under certain circumstances, the later is accessible directly in experiments. For instance, when studying the solvation of a water molecule with liquid water, the density $N(r)$ in our formulation gives the density of oxygen nuclei given a fixed location for one water molecule. As observed by Percus ${ }^{22}$, the spherical average of $N(r)$ thus corresponds to the oxygen-oxygen pair distribution function $g_{O O}(r)$ measured in experiments. Figure 3 shows our results for this quantity. In good agreement with both x-ray and neutron scattering experiments ${ }^{23}$, we find $N(r)$ to be essentially zero until a radius of $\sim 2.0 \AA$, at which point the density rises rapidly, overshooting the bulk density before finally approaching it. The experiments do show a much more pronounced peak and much more structure in the form of oscillations which occur beyond $2.5 \AA$. We believe that these discrepancies are due to the over simplified model we are using for $A_{l q}[N(r)]$ in (8) and that a better liquid density-functional would improve this.

Generally, the coordination number for a liquid is defined as the integral of $g_{O O}$ from $r=0$ to the location of the first minimum after the coordination peak. In our case there are no such oscillations. However, if we define the coordination number as the integral of $g_{O O}$ up to $3.6 \AA$, the last point before the bulk value of unity is obtained, we find a coordination of 5.3, relatively close to the value of approximately four measured in liquid water and far from the close packed value near twelve typically found in simple fluids. Thus, our model, as simple as it is, captures enough of the physics of water to reflect the hydrogen bonding network which leads to tetrahedral coordination. The model also appears to reflect the correct energetics and to give a correct ab initio prediction of the boundary position of the cavity outside of which the fluid is excluded. To our knowledge, this is the first accurate determination of such a boundary directly from first principles. As solvation energies are known to be quite sensitive to the construction of the boundary, this success gives a strong advantage to the current approach.

Finally, Figure 4 summarizes the comparison of the free energies predicted by the implementation described above with both experiment and the predictions of state-of-the-art continuum solvation models. Our joint density-functional theory results are clearly superior to dielectric-cavity-only calculations and are arguably better than state-of-the-art continuum methods that include cavity corrections. It is satisfying to see that, without any fitting or ad hoc adjustments, the hydrophobicity of methane is predicted correctly. It is also quite encouraging to see that the value we predict for the most basic test, water in water, falls much closer than do the quantum chemical methods to the correct value for inserting a water molecule at a fixed location into liquid water. While improvements and further studies are needed, the quality of these results without adjustment of parameters is very encouraging.

Although the results of the above preliminary implementation have the advantage of involving no direct fitting to solvation energies and are of comparable quality 


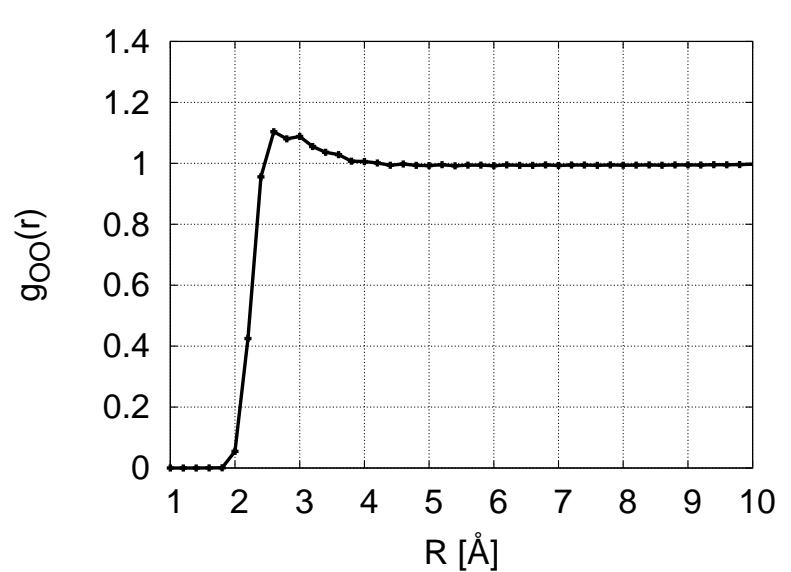

FIG. 3: Spherical average of $N(r)$ about the oxygen nucleus of an explicit water molecule in solution scaled to the bulk liquid density, corresponding to the correlation function $g_{O O}(r)$ measured in experiments on liquid water.

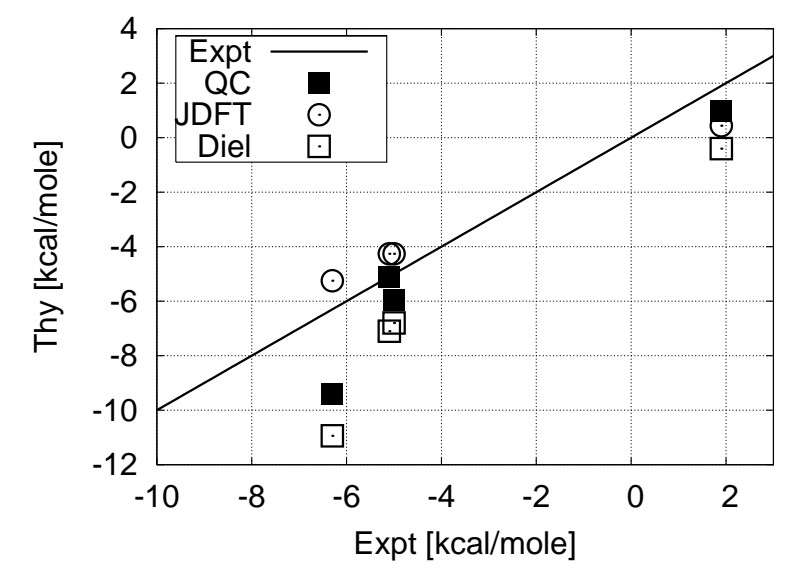

FIG. 4: Computed (vertical axis) versus experimental (horizontal axis) solvation energies for water, ethanol, methanol and methane (from left to right): perfect agreement (diagonal line), published quantum chemistry values $\left({ }^{24}\right.$ for all but water ${ }^{25}$ for water) with dielectric contribution only (open squares) and including cavitation terms (closed squares), preliminary results from joint density functional theory (open circles). to the state-of-the-art, there are a number of weaknesses in the preliminary implementation, namely (1) the lack of orientational dependence in the pseudopotential, (2) an artificial separation of the dielectric response from the internal orientational correlations of the liquid, (3) an over simplified, linear description of the dielectric response with an ad hoc nonlocal length scale, (4) the resulting need to artificially prevent the solvent from overlapping the nuclei. All of these can be addressed by a formulation including some information describing orientational ordering in the fluid, which would allow for the use of orientation-dependent pseudopotentials and could be used to more naturally describe the dielectric response with its non-local and non-linear effects. Development of such an approach is the subject of future work.
1 M. Payne, M. Teter, D. Allan, T. Arias, and J. Joannopoulos, Rev. Mod. Phys. 64, 1045 (1992).

2 J. Seminario, Recent Developments and Applications of Modern Density Functional Theory (Elsevier, Amsterdam, 1996).

3 J. Tomasi and M. Persico, Chem. Rev. 94, 2027 (1994).

4 J. Tomasi, B. Menucci, R. Cammi, and M. Cossi, in Theoretical Aspects of Biochemical Reactivity, edited by G. Naray-Szabo and A. Warshel (Kluwer, Dordrecht, 1997), p. 1.

5 J.-L. Rivail and D. Rinaldi, in Computational Chemistry, Review of Current Trends, edited by J. Leszczynski (World
Scientific, New York, 1995), p. 139.

${ }^{6}$ C. J. Cramer and D. G. Truhlar, Chem. Rev. 99, 2161 (1999).

7 F. J. Luque, C. Curutchet, J. M. noz Muriedas, A. BidonChanal, I. Soteras, A. Morreale, J. Gelpi, and M. Orozco, Phys. Chem. Chem. Phys. 5, 3827 (2003).

8 J. Dzubiella, J. M. J. Swanson, and J. A. McCammon, Phys. Rev. Lett. 96, 087802 (2006).

9 M. Young, B. Jayaram, and D. Beveridge, J. Phys. Chem. B 102, 7666 (1998).

10 N. D. Mermin, Phys. Rev. 137, A1441 (1965).

11 J. Capitani, R. Nalewajski, and R. Parr, J. Chem. Phys. 
76, 568 (1982)

12 M. Levy, Proceedings of the National Academy of Sciences of the United States of America 76, 6062 (1979).

13 T. Gilbert, Phys. Rev. B 12, 2111 (1975).

14 N. Vaidehi, T. Wesolowski, and A. Warshel, J. Chem. Phys. 97, 4264 (1992).

15 K. S. Kim, I. Park, S. Lee, K. Cho1, J. Y. Lee, J. Kim, and J. D. Joannopoulos, Phys. Rev. Lett. 76, 956 (1996).

16 I. Park, K. Cho, S. Lee, K. Kim, and J. Joannopoulos, Computational Materials Science 21, 291 (2001).

17 S. X. Sun, Phys. Rev. E 64, 021512 (2001).

18 T. F. Meister and D. M. Kroll, Phys. Rev. A 31, 4055 (1985).

19 R. Ramirez and D. Borgis, J. Phys. Chem. 109, 6754
(2005).

20 J. K. Percus and G. J. Yevick, Phys. Rev. 110, 1 (1957).

21 P. R. ten Wolde, S. X. Sun, and D. Chandler, Phys. Rev. A 137, 1441 (1965).

22 J. K. Percus, Phys. Rev. Lett. 8, 462 (1962).

${ }^{23}$ G. Hura, J. M. Sorenson, R. M. Glaeser, and T. HeadGordon, Journal of Chemical Physics 113, 9140 (2000).

24 B. Marten, K. Kim, C. Cortis, R. Friesner, R. Murphy, M. Ringnalda, D. Sitkoff, and B. Honig., J. Phys. Chem. 100, 11775 (1996).

25 T. Truong and E. Stefanovich, Chem. Phys. Lett. 240, 253 (1995). 